\title{
$\angle$ Research Square \\ Selocalcitol: Scope of inhalational formulation for prevention and cure of COVID-19 illness
}

\section{Sumanpreet Kaur}

PGIMER, Chandigarh 160012

\section{Deepak Yadav}

PGIMER, Chandigarh 160012

Sukhpreet Singh

PGIMER, Chandigarh 160012

\section{Monu Kumari}

PGIMER, Chandigarh 160012

\section{Deepak Kumar}

PGIMER, Chandigarh 160012

\section{Kirtimaan Syal}

Birla Institute of Technology and Science-Pilani, Hyderabad campus, Hyderabad, Telangana, India 500078

\section{Rajasri Bhattacharyya ( $\nabla$ bdr.rajasri@yahoo.in )}

PGIMER, Chandigarh 160012

Dibyajyoti Banerjee ( $\nabla$ dibyajyoti5200@yahoo.co.in )

PGIMER, Chandigarh 160012 https://orcid.org/0000-0003-1690-8139

\section{Research Article}

Keywords: COVID-19, Selocalcitol, Vitamin D, ACE2, VDR, Renin

Posted Date: August 24th, 2020

DOI: https://doi.org/10.21203/rs.3.rs-63402/v1

License: (c) (i) This work is licensed under a Creative Commons Attribution 4.0 International License.

Read Full License 


\section{Abstract}

Vitamin D has generated interest in the context of COVID-19 pandemic. Some authorities believe that it is beneficial; others think that without proven deficiency it should not be administered. The results of clinical trial are still awaited. Therefore nothing regarding the matter is conclusively known. In this context we believe that selocalcitol, a Vitamin $D$ analogue if administered via inhalational route, has the potential to prevent SARS-CoV2 infection. The basis of this idea is presented here.

\section{Introduction}

The world is now facing the first corona viral pandemic. [1-4] Recently discovered SARS-CoV2 causes it. [2] The disease is named as COVID-19. [2] It ranges from asymptomatic illness to a severe form of the disease. [5-6] The death toll is considerably high, and at the present moment apart from the Russian vaccine, there is no approved medication or vaccine for prevention or cure of COVID-19 illness. [7-8] Mankind has masked its face and maintaining distance to prevent viral transmission. [9] SARS-CoV2 is recognized as a respiratory pathogen. The viral S-protein binds to Angiotensin-converting receptor 2 located at bronchial epithelial cells. [10-11] After binding the ACE-2 is downregulated and then virus enters the host cell. [10] ACE2 is an isoform of Angiotensin-Converting Enzyme(ACE). [12] ACE catalyzes the formation of angiotensin II from angiotensin I. [12] On the other hand ACE2 catalyzes the breakdown of Angiotensin I to beneficial peptides. [13-14] The virus binding alters the ratio of ACE to ACE2. [15] Following virus binding, ACE2 function becomes less compared to ACE function. [15] Therefore, angiotensin II formation may increase. [14-15] Angiotensin II is a pulmonary vasoconstrictor. [16] So, the COVID-19 illness can generate severe respiratory symptoms leading to fatality. [17-19]

There is considerable current interest in Vitamin D status and COVID-19 illness. [20-21] Many authorities believe that vitamin D deficiency is related to COVID-19 illness. [22-23] We believe that Vitamin D has a paradoxical relationship with COVID-19 illness. Vitamin D inhibits renin to form less angiotensin I (the precursor of angiotensin II). [24-25] So, Vitamin D indirectly inhibits the formation of angiotensin II. [26] Vitamin D inhibits ACE as well. [27] Further Vitamin D expresses ACE2 in host cells. [28] This also helps to overcome the ill effects of inhibition of ACE2 function due to virus binding. Nevertheless, vitamin D induced overexpression of ACE2 will also invite the viral infection as the virus binds to ACE2 prior entry to the host cell. Therefore, using tools of computational biology we have searched some vitamin $D$ analogues that bind better with VDR and ACE2 in comparison to Vitamin D. Such molecule is expected to overexpress ACE2, but the virus will not get chance to bind with the protein as the analogue will preoccupy the virus binding site.

\section{Methodology}


3D Structures of Vitamin D (25-hydroxy cholecalciferol) (Pubchem id-5280453) and its analogues such as Paricalcitol (Pubchem id-5281104), Doxercalciferol (Pubchem id-5281107), Falecalciferol (Pubchem id-5282190), Maxacalcitol (Pubchem id-6398761), Tacalcitol (Pubchem id-5283734), Calcipotriol (Pubchem id-5288783), Alfacalcidol (Pubchem id-66577031), Eldecalitol (Pubchem id-6918141), Selocalcitol (Pubchem id-5288149), Lexacalcitol (Pubchem id-5288670) and Inecalcitol (Pubchem id6915835) were obtained from Pubchem database (https://pubchem.ncbi.nlm.nih.gov/). The structures of ACE2 (PDB id-6LZG) and VDR (PDB id-1DB1) proteins were downloaded from Protein Data Bank (PDB) (https://www.rcsb.org/). To screen the interactions at first vitamin $D$ and the aforementioned analogues were docked using AUTODOCK 4.2.6 with the known ligand binding sites of ACE2. The analogue that performed better in terms of binding energy was chosen for further studies.

The screened analogue and vitamin D were docked with VDR and ACE2 in separate runs for 6 times using AUTODOCK 4.2.6 and GOLD 5.3.0 software to obtain free energy of binding and the binding score. The interaction of the analogue at a known ligand-binding site is validated by Molecular Dynamics simulation run for 10 ns using GROMACS 2018.4 software following the instruction of the software manufacturer. Lys31 of ACE2 is essential for binding to SARS-CoV2. In VDR Tyr 143 and His305 are critical for ligand binding. [29-33]

The NZ atom of Lys31 from ACE2 and OH \& NE2 of Tyr143 \& His305 from VDR were chosen as the grid centre for docking.

The docked structures exhibiting the highest negative binding energy and best GOLD score with ACE2 was considered for MD simulation. In the case of VDR, docked structures at His305 were chosen. Drug topology files were generated using the SwissParam tool, and CHARMM 27 force-field was used throughout the simulation. The Protein-drug complexes were solvated in $1.2 \mathrm{~nm}$ cubic boxes of water using the TIP3P water model, and neutralization was performed using required sodium ions ( $\mathrm{Na}+)$. Energy minimization was performed for 50,000 steps using the steepest descent method. The system was then equilibrated using NVT and NPT protocols for 50,000 steps each. Then the entire system was considered for MD simulation of $10 \mathrm{~ns}$ runs at $300 \mathrm{~K}$ temperature and 1 bar pressure and results were visualized using Pymol. Xmgrace plotting tool was used to analyze the graphs of potential energy, root mean square deviation (RMSD), radius of gyration, hydrogen bonds, and other parameters as discussed in the results.

\section{Results}

In the screening study, Selocalcitol has shown most favourable binding energy with Lys31 of ACE2 compared to 25-hydroxy cholecalciferol (Vitamin D) or other studied Vitamin D analogues (Table 1).

Selocalcitol has shown significantly more $(p<0.0001)$ favourable binding free energy compared to Vitamin D when docked with ACE2 ( $n=60)$ (Table 2). When the same in-silico experiment is repeated using GOLD software, the analogue has shown significantly $(p<0.0001)$ better binding score (or Gold score) when compared to Vitamin D. The above observation is true for VDR when the grid centre of docking was 
His305 in both Autodock and GOLD. However, when Tyr143 was considered as grid centre, the similar pattern was observed with GOLD. The mean \pm SD of the observed binding energy values and GOLD score are given in Table $2 \& 3$. Binding orientations of the Vitamin $D$ and Selocalcitol molecules after docking with ACE2 and VDR are shown in Figure $1 \& 2$. In all the protein-drug complexes, at least one hydrogen bond is observed. In addition to the hydrogen bond(s), non- bonded interactions were also visualized.

The MD simulation run of the docked complex of ACE2 bound with selocalcitol in autodock showed stable binding. The potential and kinetic energy of the complex were stable throughout the simulation run. The radius of gyration decreases with time, rmsd stabilizes after $5 \mathrm{~ns}$ run, and on average one hydrogen bond is observed throughout the run (Figure 3). Similar results were obtained when docked pdb file generated using GOLD was used for MD simulation (Figure 4).

In the case of VDR, Selocalcitol docked at His305 site was used for MD simulation. At VDR as well, selocalcitol has shown stable interaction throughout the run time (Figure $5 \& 6$ )

\section{Discussion}

Authorities believe that Vitamin D is beneficial for the management of COVID-19 illness. [34]. Opposing views are also present. [35] Amidst these two divergent views, we believe that Vitamin D exhibits a paradoxical relation with COVID-19 illness. [20] Vitamin D inhibits renin. It will reduce the production of angiotensin II. It is going to be beneficial. On the other hand, Vitamin D expresses ACE2. It will cause SARS - CoV 2 infection as the virus binds with ACE2. Therefore, Vitamin D analogues may be beneficial than Vitamin D per se in COVID-19 illness if it inhibits renin and simultaneously preoccupies the virus binding site at ACE2. It is in this context our observed results are important.

We have predicted that selocalcitol binds with VDR and ACE2 better than Vitamin D. Vitamin D binding with VDR inhibits renin gene expression. [36-37, 24] We have also predicted that selocalcitol binds with VDR better than vitamin $D$. This is worth validating experimentally. If our prediction comes to be true by experimental studies then; selocalcitol will inhibit renin action better than vitamin D.

Further, in-silico selocalcitol has occupied the virus binding site of ACE2 (i.e. Lys31) better than vitamin D. So, selocalcitol may inhibit the virus binding with the ACE 2. SARS-CoV 2 binding with ACE2 is critical for the COVID-19 illness. So selocalcitol if blocks the virus binding site is expected to prevent virus binding if supplied before the virus exposure. Additionally, inhibition of the renin expression is expected to reduce pulmonary complications of COVID-19 illness. Based on the above reasons we strongly recommend experimental verification of our prediction, and if proved to be valid, we believe that an inhalational formulation of selocalcitol can be thought as a vaccination strategy for prevention of COVID19 illness.

\section{Declarations}

\section{Acknowlegement}


SK, DY and DB acknowledge PGIMER, Chandigarh, for financial assistance. DK and MK acknowledges CSIR, New Delhi, India, for providing fellowship (File No: 09/141 (0197)/2016-EMR-I, File No: 09/141 (0217)/2019-EMR-I). SS acknowledges UGC, India, for providing fellowship (F. No. 16-6(Dec. 2017)/2018(NET/CSIR).

\section{Conflict of Interest}

None

\section{References}

1. Timeline: WHO's COVID-19 response [Internet]. [cited 2020 Aug 18]. Available from: https://www.who.int/emergencies/diseases/novel-coronavirus-2019/interactive-timeline

2. Hamid S, Mir MY, Rohela GK. Novel coronavirus disease (COVID-19): a pandemic (Epidemiology, pathogenesis and potential therapeutics). New Microbes New Infect. 2020 May;35:100679.

3. COVID-19: The first documented coronavirus pandemic in history. Biomedical Journal [Internet]. 2020 May 5 [cited 2020 Aug 18]; Available from: https://www.sciencedirect.com/science/article/pii/S2319417020300445

4. Frutos R, Lopez Roig M, Serra-Cobo J, Devaux CA. Covid-19: the conjunction of events leading to the coronavirus pandemic and lessons to learn for future threats. Front Med [Internet]. 2020 [cited 2020 Aug 18];7. Available from: https://www.frontiersin.org/articles/10.3389/fmed.2020.00223/full\#B1

5. Yang P, Liu P, Li D, Zhao D. Corona Virus Disease 2019, a growing threat to children? J Infect. 2020 Jun;80(6):671-93.

6. Prevalence and severity of corona virus disease 2019 (COVID-19): A systematic review and metaanalysis. Journal of Clinical Virology. 2020 Jun 1;127:104371.

7. Thorp HH. A dangerous rush for vaccines. Science [Internet]. 2020 Aug 13 [cited 2020 Aug 18]; Available from: https://science.sciencemag.org/content/early/2020/08/12/science.abe3147

8. Coronavirus disease (COVID-19) - world health organization [Internet]. [cited 2020 Aug 18]. Available from: https://www.who.int/emergencies/diseases/novel-coronavirus-2019

9. Advice for the public on covid-19 - world health organization [Internet]. [cited 2020 Aug 18]. Available from: https://www.who.int/emergencies/diseases/novel-coronavirus-2019/advice-forpublic

10. Weiss SR, Navas-Martin S. Coronavirus pathogenesis and the emerging pathogen severe acute respiratory syndrome coronavirus. Microbiol Mol Biol Rev. 2005 Dec;69(4):635-64.

11. Jia HP, Look DC, Shi L, Hickey M, Pewe L, Netland J, et al. Ace2 receptor expression and severe acute respiratory syndrome coronavirus infection depend on differentiation of human airway epithelia. $J$ Virol. 2005 Dec;79(23):14614-21.

12. Coates $D$. The angiotensin converting enzyme (Ace). The International Journal of Biochemistry \& Cell Biology. 2003 Jun 1;35(6):769-73. 
13. Tikellis C, Thomas MC. Angiotensin-converting enzyme 2 (Ace2) is a key modulator of the renin angiotensin system in health and disease [Internet]. International Journal of Peptides. 2012 [cited 2020 Aug 18]. Available from: https://www.hindawi.com/journals/ijpep/2012/256294/

14. Turner AJ, Hiscox JA, Hooper NM. ACE2: from vasopeptidase to SARS virus receptor. Trends in Pharmacological Sciences. 2004 Jun 1;25(6):291-4.

15. Pagliaro P, Penna C. Ace/ace2 ratio: a key also in 2019 coronavirus disease (Covid-19)? Front Med [Internet]. 2020 [cited 2020 Aug 18];7. Available from: https://www.frontiersin.org/articles/10.3389/fmed.2020.00335/full

16. Kanaide Hideo, Ichiki Toshihiro, Nishimura Junji, Hirano Katsuya. Cellular mechanism of vasoconstriction induced by angiotensin ii. Circulation Research. 2003 Nov 28;93(11):1015-7.

17. Wong MKS. Subchapter 29b - angiotensin ii. In: Takei Y, Ando H, Tsutsui K, editors. Handbook of Hormones [Internet]. San Diego: Academic Press; 2016 [cited 2020 Aug 18]. p. 258-e29B-4. Available from: http://www.sciencedirect.com/science/article/pii/B978012801028000177X

18. Kuba K, Imai Y, Penninger JM. Angiotensin-converting enzyme 2 in lung diseases. Curr Opin Pharmacol. 2006 Jun;6(3):271-6.

19. Miesbach W. Pathological role of angiotensin ii in severe covid-19. TH Open. 2020 Jun 26;4(2):e13844.

20. Kumar D, Gupta P, Banerjee D. Letter: does vitamin D have a potential role against COVID-19? Alimentary Pharmacology \& Therapeutics. 2020;52(2):409-11.

21. Martineau AR, Forouhi NG. Vitamin D for COVID-19: a case to answer? The Lancet Diabetes \& Endocrinology [Internet]. 2020 Aug 3 [cited 2020 Aug 18];0(0). Available from: https://www.thelancet.com/journals/landia/article/PIIS2213-8587(20)30268-0/abstract

22. Biesalski HK. Vitamin D deficiency and co-morbidities in COVID-19 patients - A fatal relationship? Nfs Journal. 2020 Aug;20:10-21.

23. WHO I Vitamin D for prevention of respiratory tract infections [Internet]. WHO. [cited 2020 Aug 18]. Available from: http://www.who.int/elena/titles/commentary/vitamind_pneumonia_children/en/

24. Ajabshir S, Asif A, Nayer A. The effects of vitamin D on the renin-angiotensin system. $J$ Nephropathol. 2014 Apr 1;3(2):41-3.

25. Li YC. Vitamin D regulation of the renin-angiotensin system. Journal of Cellular Biochemistry. 2003;88(2):327-31.

26. Are vitamin $D$ receptor agonists like angiotensin-converting enzyme inhibitors without side effects? Kidney International. 2010 Jun 1;77(11):943-5.

27. Ali RM, Al-Shorbagy MY, Helmy MW, El-Abhar HS. Role of Wnt4/ $\beta$-catenin, Ang II/TGF $\beta$, ACE2, NF-kB, and IL-18 in attenuating renal ischemia/reperfusion-induced injury in rats treated with Vit $D$ and pioglitazone. European Journal of Pharmacology. 2018 Jul 15;831:68-76.

28. Zwart SR, Smith SM. Vitamin d and covid-19: lessons from spaceflight analogs. J Nutr [Internet]. [cited 2020 Aug 18]; Available from: https://academic.oup.com/jn/advance- 
article/doi/10.1093/jn/nxaa233/5876210

29. Wan Y, Shang J, Graham R, Baric RS, Li F. Receptor recognition by the novel coronavirus from wuhan: an analysis based on decade-long structural studies of sars coronavirus. Journal of Virology [Internet]. 2020 Mar 17 [cited 2020 Aug 18];94(7). Available from:

https://jvi.asm.org/content/94/7/e00127-20

30. ACE2 receptor polymorphism: Susceptibility to SARS-CoV-2, hypertension, multi-organ failure, and COVID-19 disease outcome. Journal of Microbiology, Immunology and Infection. 2020 Jun 1;53(3):425-35.

31. Ribone SR, Ferronato MJ, Vitale C, Fall Y, Curino AC, Facchinetti MM, et al. Vitamin D receptor exhibits different pharmacodynamic features in tumoral and normal microenvironments: A molecular modeling study. The Journal of Steroid Biochemistry and Molecular Biology. 2020 Jun 1;200:105649.

32. Takeda R, Kobayashi I, Suzuki R, Kawai K, Kittaka A, Takimoto-Kamimura M, et al. Proposal of potent inhibitors for vitamin-D receptor based on ab initio fragment molecular orbital calculations. Journal of Molecular Graphics and Modelling. 2018 Mar 1;80:320-6.

33. Zheng J, Chang MR, Stites RE, Wang Y, Bruning JB, Pascal BD, et al. HDX reveals the conformational dynamics of DNA sequence specific VDR co-activator interactions. Nature Communications. 2017 Oct 13;8(1):1-13.

34. Martineau AR, Forouhi NG. Vitamin D for COVID-19: a case to answer? The Lancet Diabetes \& Endocrinology [Internet]. 2020 Aug 3 [cited 2020 Aug 18];0(0). Available from: https://www.thelancet.com/journals/landia/article/PIIS2213-8587(20)30268-0/abstract

35. Trovas G, Tournis S. Vitamin d and covid-19. Hormones (Athens). 2020 Jul 14;1-2.

36. Vaidya A, Williams JS. The relationship between vitamin $D$ and the renin-angiotensin system in the pathophysiology of hypertension, kidney disease, and diabetes. Metabolism - Clinical and Experimental. 2012 Apr 1;61(4):450-8.

37. Sigmund CD. Regulation of renin expression and blood pressure by vitamin D3. J Clin Invest. 2002 Jul 15;110(2):155-6.

\section{Tables}

Table 1- Binding Energy values of Vitamin D and its analogues docked at Lys31 of ACE2 with Autodock. 


\begin{tabular}{|l|l|l|}
\hline Sr. No & Drug & Highest binding energy (Kcal/mol) at Lys31 of ACE2 \\
\hline 1 & Vitamin D & -5.77 \\
\hline 2 & Paricalcitol & -5.94 \\
\hline 3 & Doxercalciferol & -6.52 \\
\hline 4 & Falecalcitriol & -5.42 \\
\hline 5 & Maxacalcitol & -6.14 \\
\hline 6 & Tacalcitol & -6.03 \\
\hline 7 & Calcipotriol & -6.07 \\
\hline 8 & Alfacalcidol & -6.43 \\
\hline 9 & Eldecalcidol & -4.94 \\
\hline 10 & Selocalcitol & -6.79 \\
\hline 11 & Lexacalcitol & -5.24 \\
\hline 12 & Inecalcitol & -6.48 \\
\hline
\end{tabular}

Table 2- Binding energy (Kcal/mol) and GOLD score of Vitamin D and Selocalcitol docked at Lys31 of ACE2 with Autodock and GOLD.

\begin{tabular}{|l|l|l|}
\hline Drug & Mean \pm SD (Autodock) & Mean \pm SD (GOLD) \\
\hline Vitamin D & $-5.1 \pm 0.60(\mathrm{n}=60)$ & $34.18 \pm 6.79(\mathrm{n}=29)$ \\
\hline Selocalcitol & $-5.81 \pm 0.67(\mathrm{n}=60)^{* * *}$ & $39.65 \pm 7.91(\mathrm{n}=31)^{* * *}$ \\
\hline
\end{tabular}

$* * * \mathrm{p}$ value $<0.0001$

Table 3- Binding energy (Kcal/mol) and GOLD score of Vitamin D and Selocalictol docked at various active sites (Tyr143 and His305) of VDR with Autodock and GOLD.

\begin{tabular}{|l|l|l|l|}
\hline Software & Drugs & Tyr143 & His305 \\
\hline \multirow{2}{*}{ AUTODOCK } & Vitamin D & $-7.85 \pm 0.67(\mathrm{n}=60)$ & $-9.89 \pm 0.46(\mathrm{n}=60)$ \\
\cline { 2 - 4 } & Selocalcitol & $-6.81 \pm 0.81(\mathrm{n}=60)$ & $-10.49 \pm 0.67(\mathrm{n}=60)^{* * *}$ \\
\hline \multirow{2}{*}{ GOLD } & Vitamin D & $44.18 \pm 8.19(\mathrm{n}=50)$ & $47.25 \pm 4.58(\mathrm{n}=50)$ \\
\cline { 2 - 4 } & Selocalcitol & $52.52 \pm 5.83(\mathrm{n}=39)^{* * *}$ & $53.09 \pm 4.53(\mathrm{n}=47)^{* * *}$ \\
\hline
\end{tabular}

$* * *$ p value $<0.0001$

Figures 

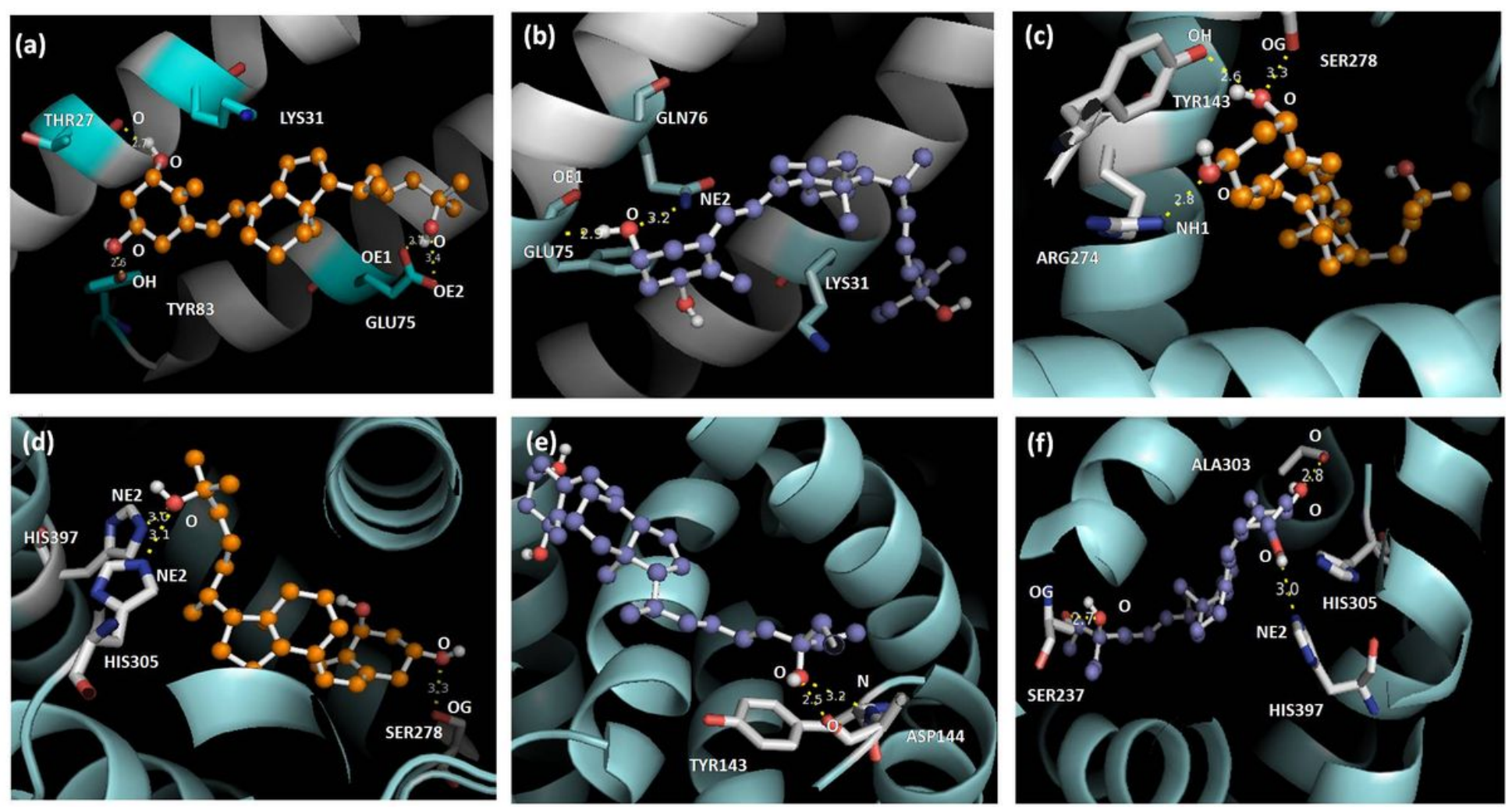

Figure 1

Docked complex (via Autodock) showing interactions of (a) Vitamin D and (b) Selocalcitol with ACE2 when Lys31 was chosen as grid centre. The interaction of Vitamin D and Selocalcitol at grid centre Tyr143 and His305 of VDR is shown in $c \& d$ (Vitamin D) and e \& $f$ (Selocalcitol) respectively. The drugs are shown in ball and stick while the protein is shown in cartoon mode. The probable hydrogen bonds are represented by yellow dotted lines along with the residue name of protein and distances are labeled. The residues involved in hydrogen bonding are shown in the stick. 

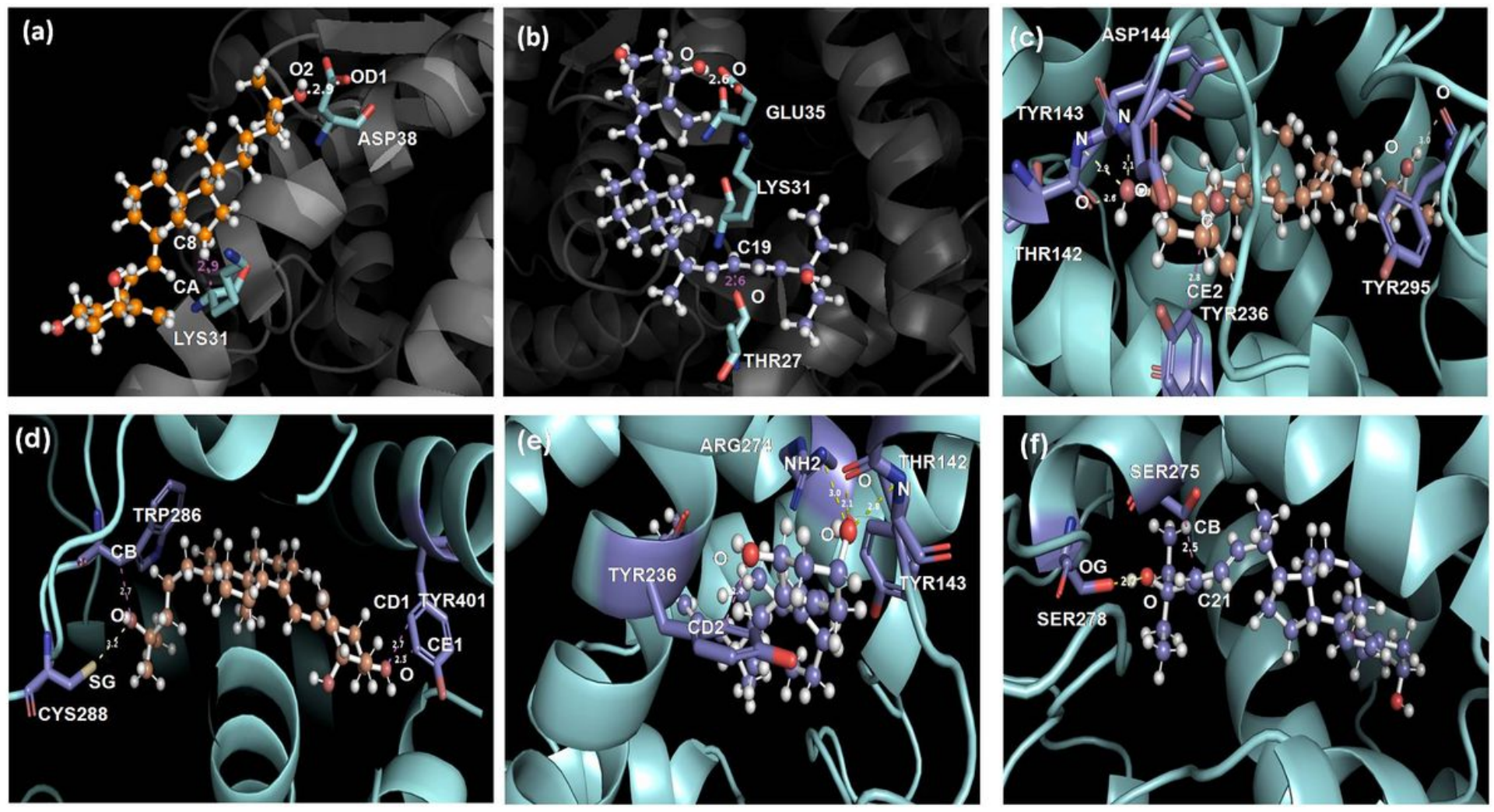

Figure 2

Docked complex (via GOLD) showing interactions of (a) Vitamin D and (b) Selocalcitol with ACE2 when Lys31 was chosen as grid centre. The interaction of Vitamin D and Selocalcitol at grid centre Tyr143 and His305 of VDR is shown in $c$ \& $d$ (Vitamin D) and e \& $f$ (Selocalcitol) respectively. The drugs are shown in ball and stick while the protein is shown in cartoon mode. The probable hydrogen bonds are represented by yellow dotted lines whereas the short contacts are presented by magenta lines along with the residue name of protein and distances are labelled. The residues involved in hydrogen bonding are shown in the stick. 

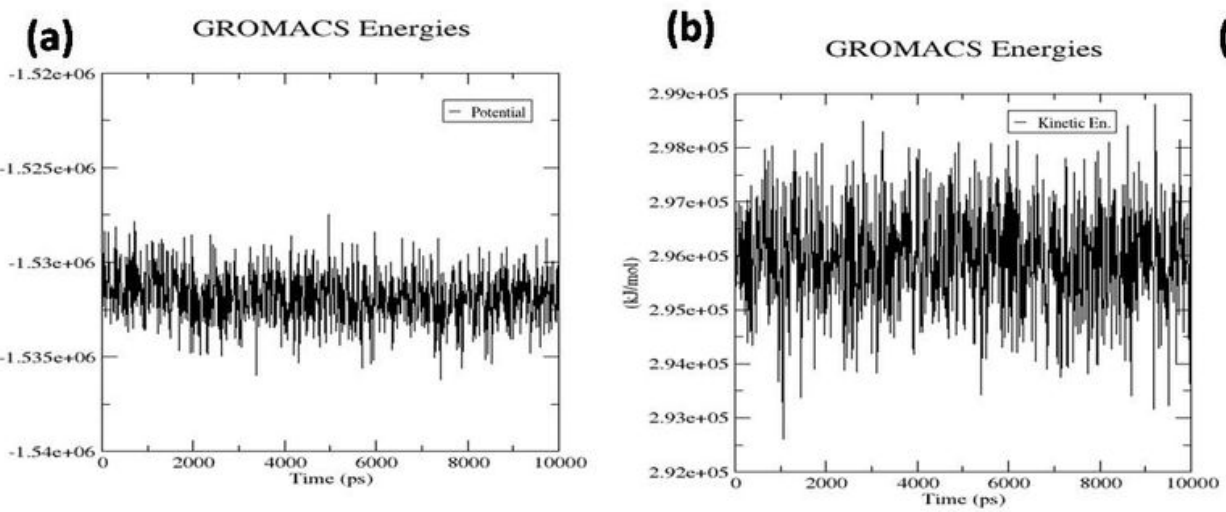

(c) Radius of gyration (total and around axes)

(d)

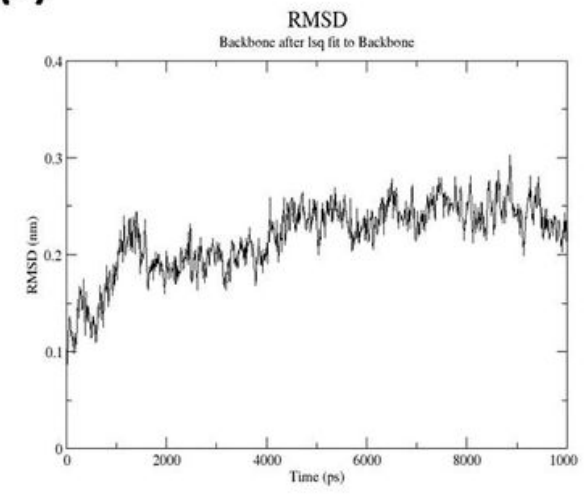

(e)
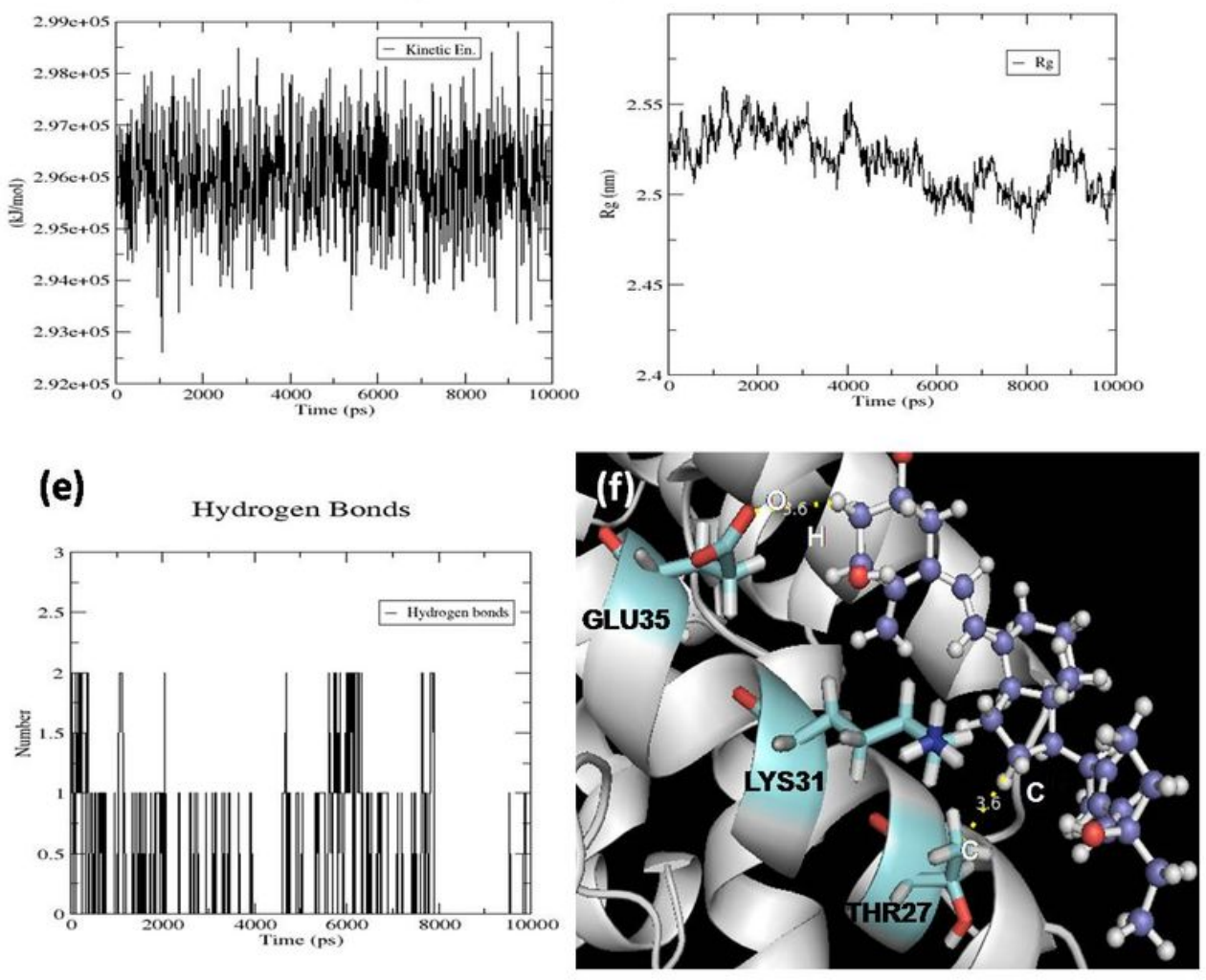

Figure 3

Molecular dynamics (MD) simulation results of the ACE2-Selocalcitol complexes (via Autodock) for $10 \mathrm{~ns}$ run are presented. The graphs represent (a) Potential energy (b) Kinetic energy (c) Radius of gyration (d) Root mean square deviation and (e) Hydrogen bonds and (f) docked structure. 

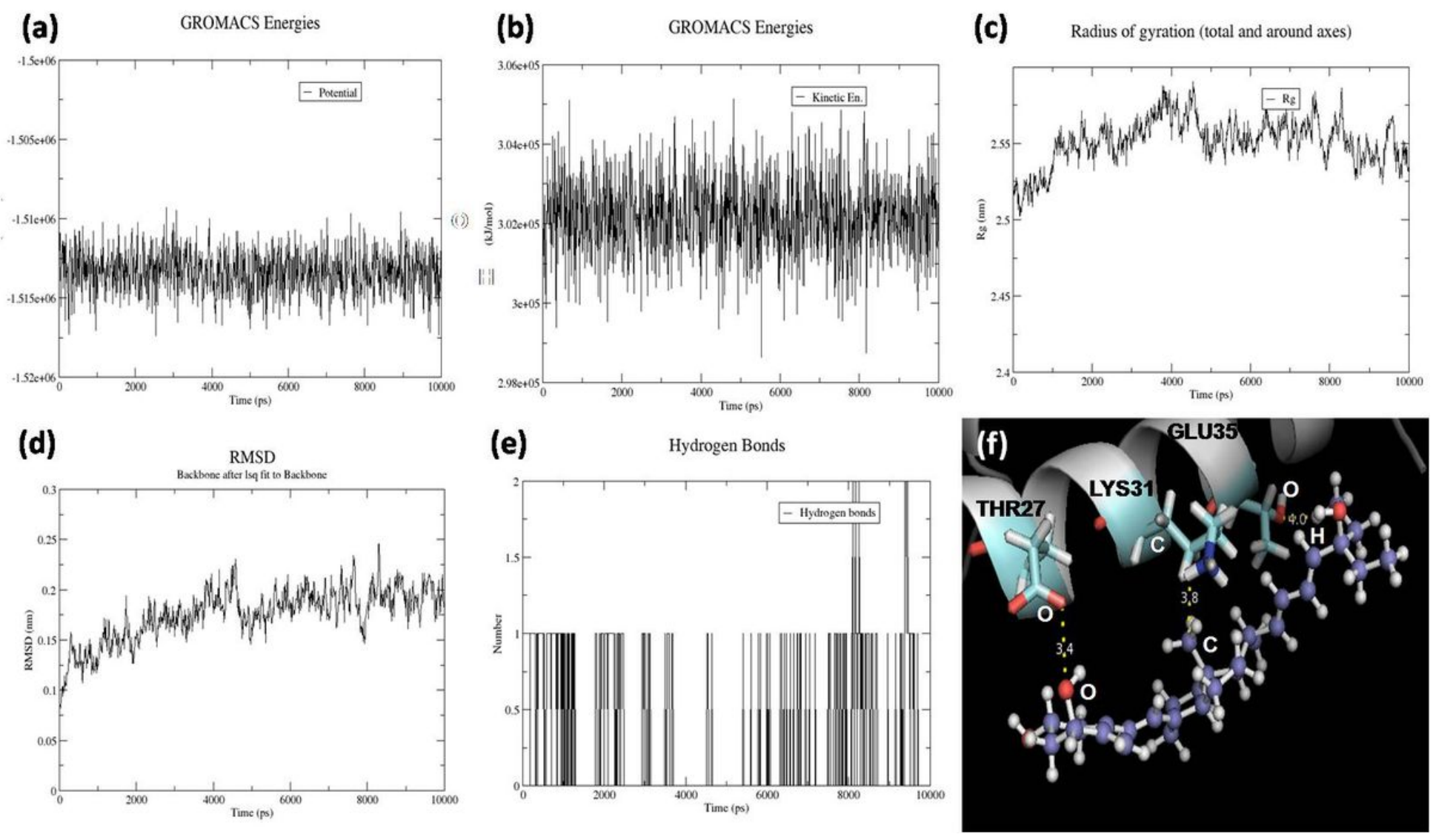

Figure 4

Molecular dynamics (MD) simulation results of the ACE2-Selocalcitol complexes (via GOLD) for $10 \mathrm{~ns}$ run are presented. The graphs represent (a) Potential energy (b) Kinetic energy (c) Radius of gyration (d) Root mean square deviation and (e) Hydrogen bonds and (f) docked structure. 
(a)

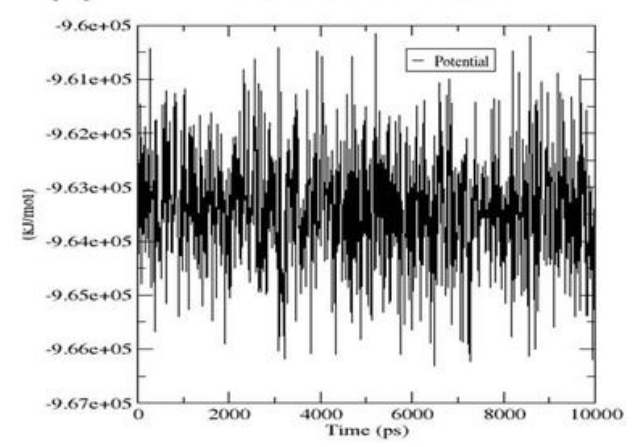

(d)

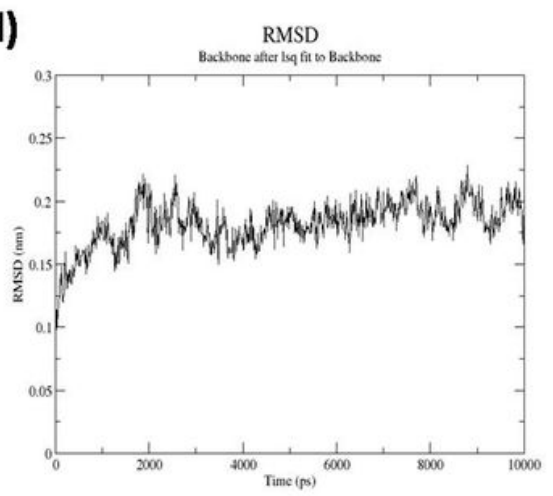

(b)

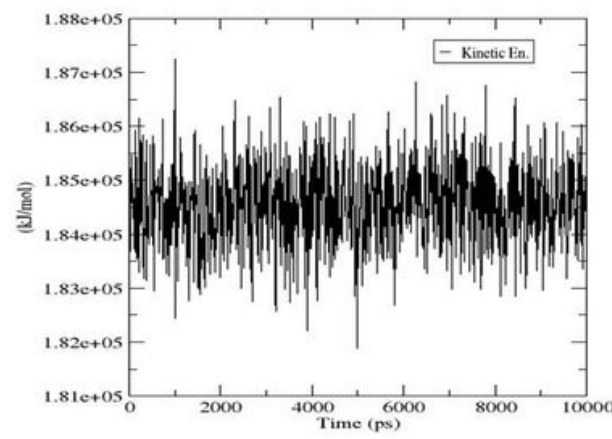

(e)

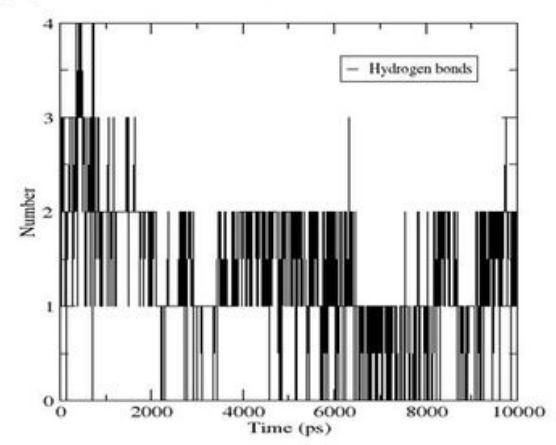

(c) Radius of gyration (total and around axes)
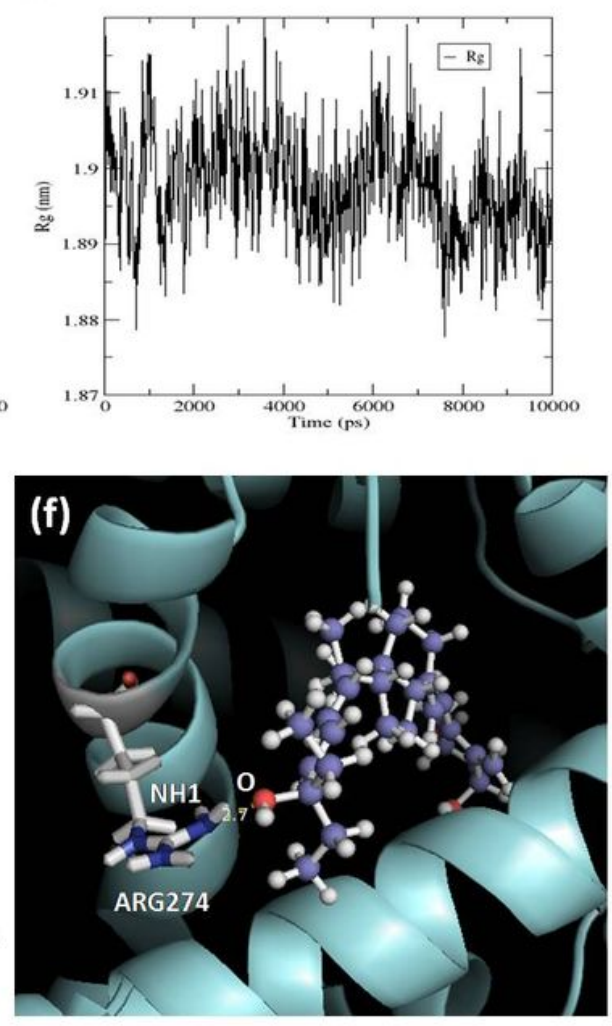

Figure 5

Molecular dynamics (MD) simulation results of the VDR-Selocalcitol complexes (via Autodock) for $10 \mathrm{~ns}$ run are presented. The graphs represent (a) Potential energy (b) Kinetic energy (c) Radius of gyration (d) Root mean square deviation and (e) Hydrogen bonds and (f) docked structure. 
(a)

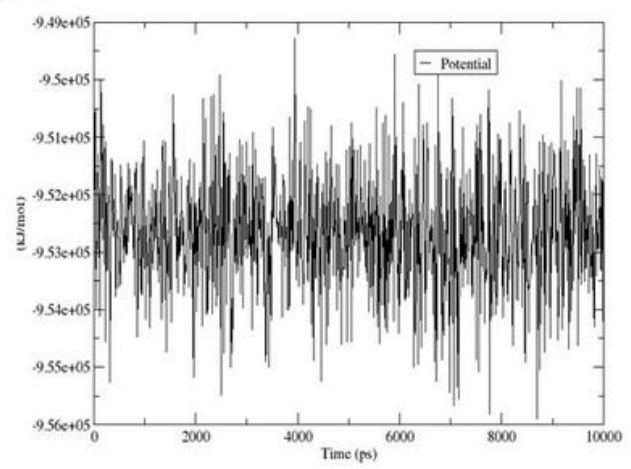

(d)

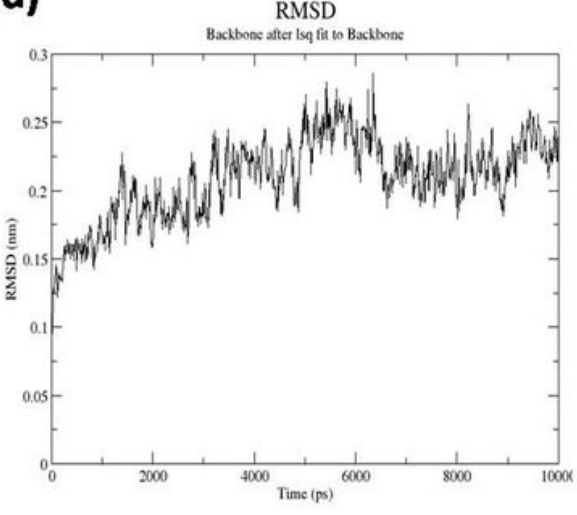

(b)

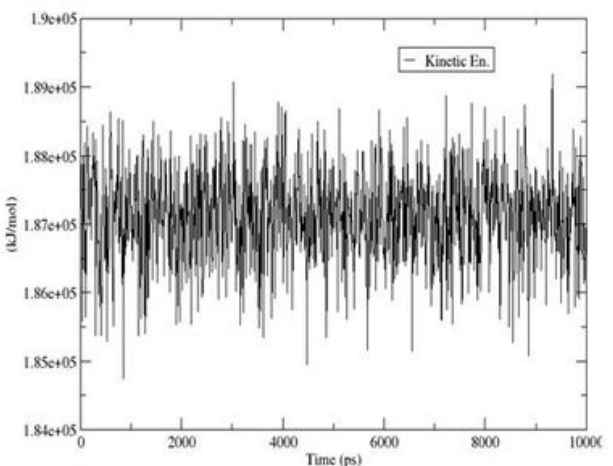

(C) Radius of gyration (total and around axes)

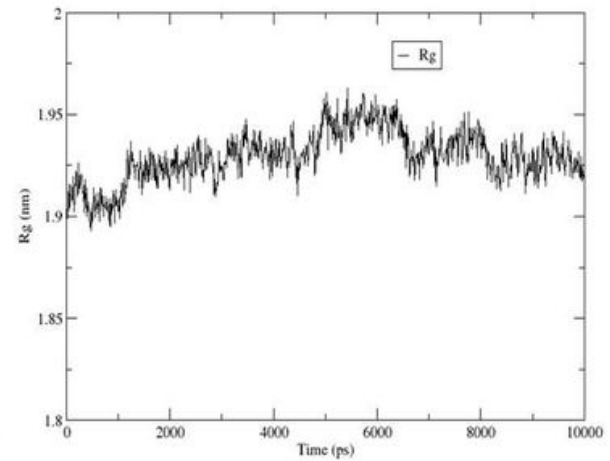

(e)

Hydrogen Bonds

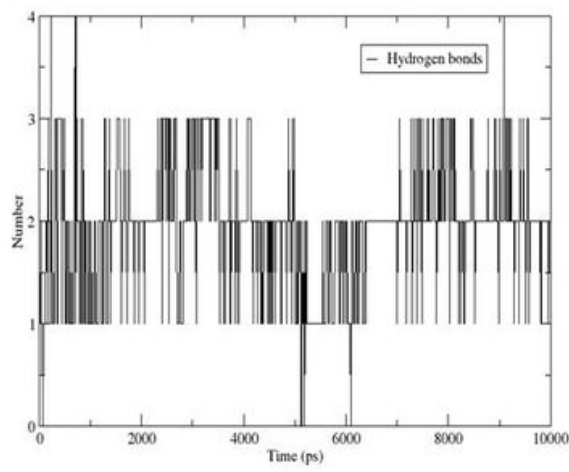

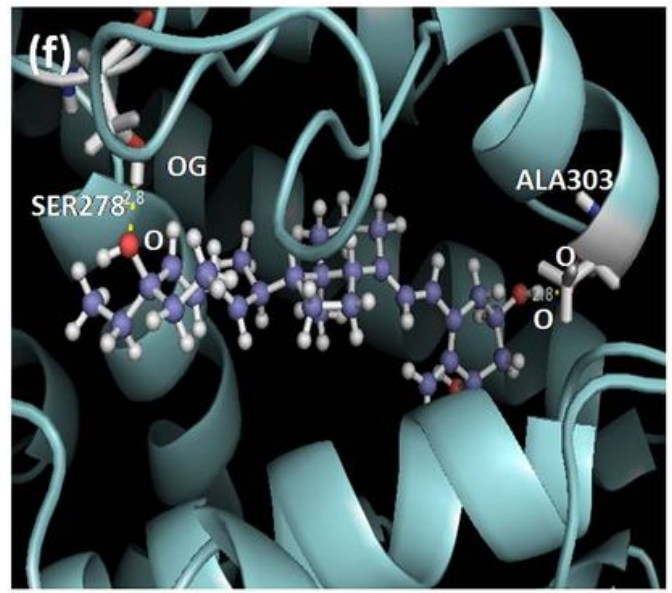

Figure 6

Molecular dynamics (MD) simulation results of the VDR-Selocalcitol complexes (via GOLD) for 10 ns run are presented. The graphs represent (a) Potential energy (b) Kinetic energy (c) Radius of gyration (d) Root mean square deviation and (e) Hydrogen bonds and (f) docked structure. 